\title{
On the spectrum of Dirac operators with the unbounded potential at infinity
}

\author{
(Dedicated to Professor Kôji Kubota on his sixtieth birthday)
}

\section{Osanobu YAMADA}

(Received April 24, 1996)

Abstract. In this paper we investigate the spectra of Dirac operators

$$
H=\sum_{j=1}^{3} \alpha_{j} D_{j}+p(x) \beta+q(x) I_{4}
$$

in the Hilbert space $\left[L^{2}\left(\mathbf{R}^{3}\right)\right]^{4}$. We show mainly that if $|p(x)| \longrightarrow \infty, q(x)=o(p(x))$ as $|x| \longrightarrow \infty$, then the spectrum of $H$ is purely discrete in the whole line $\mathbf{R}$, and if $p(x) \equiv q(x) \longrightarrow \infty$ as $|x| \longrightarrow \infty$, then the spectrum of $H$ is purely discrete in the half line $\mathbf{R}^{+}$.

Key words: Dirac operators, purely discrete spectrum.

\section{Introduction and Results}

In this paper we consider the following type of Dirac operators

$$
L=\sum_{j=1}^{3} \alpha_{j} D_{j}+p(x) \beta+q(x) I_{4}, \quad x \in \mathbf{R}^{3}, \quad D_{j}=-i \frac{\partial}{\partial x_{j}},
$$

on $\mathcal{H}=\left[L^{2}\left(\mathbf{R}^{3}\right)\right]^{4}$, where $p(x)$ and $q(x)$ are real-valued continuous functions, and

$$
\begin{aligned}
& \alpha_{j}=\left(\begin{array}{cc}
\mathbf{0} & \sigma_{j} \\
\sigma_{j} & \mathbf{0}
\end{array}\right) \quad(1 \leq j \leq 3), \quad \beta=\left(\begin{array}{cc}
I_{2} & \mathbf{0} \\
\mathbf{0} & -I_{2}
\end{array}\right), \quad I_{4}=\left(\begin{array}{cc}
I_{2} & \mathbf{0} \\
\mathbf{0} & I_{2}
\end{array}\right), \\
& \sigma_{1}=\left(\begin{array}{cc}
0 & 1 \\
1 & 0
\end{array}\right), \quad \sigma_{2}=\left(\begin{array}{cc}
0 & -i \\
i & 0
\end{array}\right), \quad \sigma_{3}=\left(\begin{array}{cc}
1 & 0 \\
0 & -1
\end{array}\right), \quad I_{2}=\left(\begin{array}{ll}
1 & 0 \\
0 & 1
\end{array}\right) .
\end{aligned}
$$

The matrices $\alpha_{j}(1 \leq j \leq 3)$ and $\alpha_{4}=\beta$ are Hermitian symmetric matrices satisfying the anti-commutation relations

$$
\alpha_{j} \alpha_{k}+\alpha_{k} \alpha_{j}=2 \delta_{j k} I_{4} \quad(1 \leq j, k \leq 4) .
$$


The symmetric operator $L$ defined on $\left[C_{0}^{\infty}\left(\mathbf{R}^{3}\right)\right]^{4}$ is essentially selfadjoint (see, e.g., Jörgens $[\amalg]]$ ). We denote the unique selfadjoint realization by $H$.

Our interest here is to investigate the spectrum of the Dirac operator $H$ satisfying

$$
|p(x)| \longrightarrow \infty, q(x)=o(p(x)) \text { as }|x| \longrightarrow \infty
$$

or

$$
p(x) \equiv q(x) \longrightarrow \infty \text { as }|x| \longrightarrow \infty .
$$

Recently, the studies of Dirac operators satisfying (2) or (3) appear in physical articles (see, e.g., Ikhdair-Mustafa-Sever [IMS], Jena-Tripati [JT], Ram-Halasa $[\mathrm{RH}])$. Although the numerical analysis of the eigenvalues is studied there, it seems that the mathematical structure of the spectrum of $H$ is not written explicitely. Their potentials are of types

$$
\begin{aligned}
& p(x)=a|x|^{2}+b, \quad q(x) \equiv 0 \quad \text { in }[\mathrm{RH}], \\
& p(x) \equiv q(x)=a|x|^{\nu}+b \quad(\nu=0.1) \text { in }[\mathrm{JT}] \text { and [IMS], } \\
& p(x) \equiv q(x)=a \log |x|+b \quad \text { in }[\mathrm{IMS}],
\end{aligned}
$$

where $a>0$ and $b$ are some real numbers.

If we assume that $p(x) \equiv 1$ and $|q(x)| \longrightarrow \infty(|x| \rightarrow \infty)$, it is shown by Titchmarsh $[\mathrm{T}]$ and Erdélyi [E] that the absolutely continuous spectrum of $H$ covers the whole line, and the singular spectrum of $H$ is empty under the condition that $q(x)=q(|x|)$ is spherically symmetric and

$$
\int_{R}^{+\infty} \frac{\left|q^{\prime}(r)\right|}{q(r)^{2}} d r<\infty \text { for some } R>0
$$

(see also Thaller [Th], Chapter 4 and Schmidt [Sc]). On the other hand, if we assume (2) or (3), we have the different structure of spectrum of $H$, which we will study in this paper.

Before we state our results, we explain some notations:

$\sigma(H)=$ the spectrum of $H$, i.e., the complement of the resolvent set of $H$, $\sigma_{p}(H)=$ the set of all the eigenvalues of $H$, $\sigma_{d}(H)=$ the set of all the isolated eigenvalues of $H$ with finite multiplicity, $\sigma_{\text {ess }}(H)=\sigma(H) \backslash \sigma_{d}(H)$, $\mathbf{R}^{+}=(0,+\infty), \quad \mathbf{R}^{-}=(-\infty, 0)$. 
Our results are as follows;

Theorem 1 Assume that $p(x) \in C^{1}$ and $q(x) \in C^{1}$ satisfy the following conditions:

$(\mathrm{a}-1)|p(x)| \longrightarrow \infty$ as $|x| \longrightarrow \infty$,

(a-2) There exist positive constants $R$ and $\varepsilon_{0}<1$ such that

$$
|q(x)| \leq \varepsilon_{0}|p(x)| \quad(|x| \geq R) .
$$

(a-3) $|\nabla p(x)|=O(p(x)), \quad|\nabla q(x)|=O(p(x))$ as $|x| \longrightarrow \infty$.

Then we have $\sigma(H)=\sigma_{d}(H)$.

Theorem 2 Assume that $p(x) \equiv q(x) \in C^{0}$ satisfies

(b-1) $q(x) \longrightarrow \infty$ as $|x| \longrightarrow \infty$.

Then we have $\sigma(H) \cap \mathbf{R}^{+}=\sigma_{d}(H)$.

Concerning the negative spectrum of $H$ under the assumption of Theorem 2, we have a result for a class of potentials $q(x)=O\left(|x|^{2}\right)$ at infinity, as follows.

Proposition 3 Assume that $p(x) \equiv q(x) \in C^{0}$ with the radial derivative satisfies

(c-1) $q(x) \longrightarrow \infty$ as $|x| \longrightarrow \infty$,

(c-2) There are positive constants $C, R$ and $1 \leq \alpha \leq 2$ such that

$$
q(x) \leq C|x|^{\alpha}, \quad \frac{2(\alpha-1)}{r} q(x) \leq \frac{\partial q}{\partial r} \quad(|x| \geq R),
$$

where $r=|x|$. Then we have $\mathbf{R}^{-} \subset \sigma_{\text {ess }}(H)$ and $\sigma_{p}(H) \cap\left(\mathbf{R}^{-} \cup\{0\}\right)=\emptyset$.

Theorem 1 will be proved in $\S 2$, and Theorem 2 in $\S 3$. The proof of Proposition 3 and some remarks will be given in $\S 4$. In Theorems 1, 2 and Proposition 3 we may allow some local singularities of $p(x)$ and $q(x)$, which we omit for the sake of simplicity.

Example. If $q(x)$ is a positive homogeneous function of degree $0<\theta \leq 2$, then $q(x)$ satisfies (c-1) and (c-2) with $\alpha=1+(\theta / 2) . q(x)=\log r$ satisfies (c-1) and (c-2) with $\alpha=1$.

\section{Proof of Theorem 1}

We prove under the assumption that the resolvent $(H-i)^{-1}$ is a compact operator in $\mathcal{H}=\left[L^{2}\left(\mathbf{R}^{3}\right)\right]^{4}$, which yields Theorem 1 . Let $\left\{f_{n}\right\}_{n=1,2, \ldots}$ be any 
bounded sequence in $\mathcal{H}$, say, $\left\|f_{n}\right\| \leq C(n=1,2, \cdots)$ for a positive constant $C$, where \|\| is the norm in $\mathcal{H}$. Then we set

$$
u_{n}=(H-i)^{-1} f_{n} \in \mathcal{H}_{l o c}^{1}:=\left[H_{l o c}^{1}\right]^{4} \quad n=1,2, \ldots,
$$

where $H_{l o c}^{1}$ is the local Sobolev space of all functions locally in the Sobolev space $H^{1}$. The sequence $\left\{u_{n}\right\}_{n=1,2, \ldots}$ clearly satisfies

$$
\left\|u_{n}\right\| \leq\left\|f_{n}\right\| \leq C \quad(n=1,2, \cdots)
$$

Let us put $P(x)=\sqrt{p(x)^{2}-q(x)^{2}} \quad(|x| \geq R)$ and $P(x)=0 \quad(|x| \leq R)$. We show below that the sequence $\left\{u_{n}\right\}_{n=1,2, \ldots}$ is bounded in a Hilbert space

$$
\mathcal{H}_{P}=\left\{g \in \mathcal{H} \mid\|g\|_{P}^{2}:=\|g\|^{2}+\|P g\|^{2}+\sum_{j=1}^{3}\left\|D_{j} g\right\|^{2}<\infty\right\}
$$

with the inner product

$$
(f, g)_{P}=(f, g)+(P f, P g)+\sum_{j=1}^{3}\left(D_{j} f, D_{j} g\right),
$$

where \|\| and $(, \quad)$ are the usual norm and the inner product in $\mathcal{H}$, respectively. The assumption $(\mathrm{a}-2)$ of Theorem 1 gives

$$
P(x)^{2}=p(x)^{2}-q(x)^{2} \leq p(x)^{2} \leq \frac{1}{1-\varepsilon_{0}^{2}} P(x)^{2} \quad(|x| \geq R) .
$$

which implies

$$
\mathcal{H}_{P}=\left\{g \in \mathcal{H} \mid\|g\|^{2}+\|p g\|^{2}+\sum_{j=1}^{3}\left\|D_{j} g\right\|^{2}<\infty\right\} .
$$

The sesquilinear forms $(f, g)_{P}$ and $(f, g)$ are also used for $f \in\left[\mathcal{D}^{\prime}\right]^{4}$ and $g \in \mathcal{D}^{4}$, where $\mathcal{D}=C_{0}^{\infty}\left(\mathbf{R}^{3}\right)$ and $\mathcal{D}^{\prime}$ is the space of distributions on $\mathbf{R}^{3}$.

Operating $\vec{\alpha} \cdot \vec{D}=\sum_{j=1}^{3} \alpha_{j} D_{j}$ to

$$
(\vec{\alpha} \cdot \vec{D}) u_{n}+p(x) \beta u_{n}+q(x) u_{n}-i u_{n}=f_{n}
$$

and using the anti-commutation relation (1) we have

$$
\begin{aligned}
-\Delta u_{n}+\left[p(x)^{2}-q(x)^{2}+1\right] u_{n}+[2 i q(x)+(\vec{\alpha} \cdot \vec{D} p) \beta+(\vec{\alpha} \cdot \vec{D} q)] u_{n} \\
=(\vec{\alpha} \cdot \vec{D}) f_{n}+[p(x) \beta-q(x)+i] f_{n} .
\end{aligned}
$$


Take a $C^{\infty}$ function $\gamma(x)$ such that $\gamma(x)=1 \quad(|x| \geq R+1)$ and $\gamma(x)=$ $0 \quad(|x| \leq R)$. For any $\psi \in \mathcal{D}^{4}$ we have

$$
\begin{aligned}
\left(\gamma u_{n}, \psi\right)_{P}= & \left(-\Delta\left(\gamma u_{n}\right)+\left[1+P(x)^{2}\right]\left(\gamma u_{n}\right), \psi\right) \\
= & \left(-(\Delta \gamma) u_{n}-2 \vec{\nabla} \gamma \cdot \vec{\nabla} u_{n}-\gamma \Delta u_{n}\right. \\
& \left.\quad+\left[1+P(x)^{2}\right]\left(\gamma u_{n}\right), \psi\right),
\end{aligned}
$$

and, by using (6),

$$
\begin{aligned}
\left(\gamma u_{n}, \psi\right)_{P}= & -\left(u_{n},(\Delta \gamma) \psi\right)+2\left(u_{n}, \vec{\nabla} \cdot[(\vec{\nabla} \gamma) \psi]\right) \\
& +\left(f_{n},(\vec{\alpha} \cdot \vec{D} \gamma) \psi\right)+\left(f_{n}, \gamma(\vec{\alpha} \cdot \vec{D}) \psi+\gamma[p \beta-q-i] \psi\right) \\
& +\left(u_{n}, \gamma[2 i q+\beta(\vec{\alpha} \cdot \vec{D} p)+(\vec{\alpha} \cdot \vec{D} q)] \psi\right) .
\end{aligned}
$$

Therefore we can find a positive constant $C_{1}$ from (4), (5) and the assumptions $(\mathrm{a}-2),(\mathrm{a}-3)$ such that

$$
\left|\left(\gamma u_{n}, \psi\right)_{P}\right| \leq C_{1}\left(\left\|f_{n}\right\|+\left\|u_{n}\right\|\right) \cdot\|\psi\|_{P} \leq 2 C C_{1}\|\psi\|_{P} \quad\left(\forall \psi \in \mathcal{D}^{4}\right) .
$$

Since $\mathcal{D}^{4}$ is dense in $\mathcal{H}_{P}$, we have $\gamma u_{n} \in \mathcal{H}_{P}$ and

$$
\left\|\gamma u_{n}\right\|_{P} \leq 2 C C_{1}, \quad n=1,2, \cdots .
$$

The above inequality and the assumption (a-1) give the relative compactness of the sequence $\left\{u_{n}\right\}_{n=1,2, \ldots}$ in $\mathcal{H}$ (see, e.g., Reed-Simon [RS], Theorem XIII.65).

\section{Proof of Theorem 2}

Let $\lambda$ be an arbitrary positive number. We show below that $\lambda$ does not belong to the essential spectrum $\sigma_{e s s}(H)$ of $H$, that is, there is no orhtonormal system $\left\{u_{n}\right\}_{n=1,2, \cdots}$ in $\mathcal{H}$ such that

$$
\left\{u_{n}\right\}_{n=1,2, \cdots} \subset D(H),\left\|H u_{n}-\lambda u_{n}\right\| \longrightarrow 0 \text { as } n \longrightarrow \infty,
$$

where $D(H)$ denotes the domain of $H$. Assume that such an orthonormal system $\left\{u_{n}\right\}_{n=1,2, \cdots}$ would exist. Then write

$$
\begin{aligned}
u_{n}=\left(\begin{array}{c}
v_{n} \\
w_{n}
\end{array}\right), \quad(H-\lambda) u_{n} & =\left(\begin{array}{c}
f_{n} \\
g_{n}
\end{array}\right), \\
& \left(v_{n}, w_{n}, f_{n}, g_{n} \in \mathbf{h}:=\left[L^{2}\left(\mathbf{R}^{3}\right)\right]^{2}\right)
\end{aligned}
$$


Then we have

$$
\begin{aligned}
& (\vec{\sigma} \cdot \vec{D}) w_{n}+2 q(x) v_{n}-\lambda v_{n}=f_{n}, \\
& (\vec{\sigma} \cdot \vec{D}) v_{n}-\lambda w_{n}=g_{n},
\end{aligned}
$$

where $(\vec{\sigma} \cdot \vec{D})=\sum_{j=1}^{3} \sigma_{j} D_{j}$. In view of Rellich's theorem we may assume that $\left\{v_{n}\right\}$ and $\left\{w_{n}\right\}$ are strongly convergent in $\left[L^{2}(\Omega)\right]^{2}$ for any bounded domain $\Omega$, by selecting a subsequence if necessary. Operating $\vec{\sigma} \cdot \vec{D}$ to (9) and using (8), we get

$$
-\Delta v_{n}+2 \lambda q(x) v_{n}=(\vec{\sigma} \cdot \vec{D}) g_{n}+\lambda f_{n}+\lambda^{2} v_{n}
$$

Take a positive number $R$ such that $q(x) \geq 1 \quad(|x| \geq R)$ by means of (b-1), and put

$$
Q(x)=\sqrt{2 \lambda q(x)}(|x| \geq R) \text { and } Q(x)=1 \quad(|x| \leq R) .
$$

Let us prepare a Hilbert space $\mathbf{h}_{Q}$ :

$$
\mathbf{h}_{Q}=\left\{g \in \mathbf{h}=\left[L^{2}\left(\mathbf{R}^{3}\right)\right]^{2} \mid\|g\|_{Q}^{2}:=\|Q g\|_{\mathbf{h}}^{2}+\sum_{j=1}^{3}\left\|D_{j} g\right\|_{\mathbf{h}}^{2}<\infty\right\}
$$

with the inner product

$$
(f, g)_{Q}=\langle Q f, Q g\rangle+\sum_{j=1}^{3}\left\langle D_{j} f, D_{j} g\right\rangle,
$$

where \|\|$_{\mathbf{h}}$ and $\langle$,$\rangle are the norm and the inner product in \mathbf{h}$, respectively. The sesquilinear forms $(f, g)_{Q}$ and $(f, g)$ are also used as in $\S 2$ for $f \in\left[\mathcal{D}^{\prime}\right]^{2}$ and $g \in \mathcal{D}^{2}$. Let $\gamma(x)$ be the same function as in $\S 2$. Then, for any $\varphi \in \mathcal{D}^{2}$ we have

$$
\begin{aligned}
\left(\gamma v_{n}, \varphi\right)_{Q} & =\left\langle-\Delta\left(\gamma v_{n}\right)+2 \lambda q\left(\gamma v_{n}\right), \varphi\right\rangle \\
& =\left\langle-(\Delta \gamma) v_{n}-2 \vec{\nabla} \gamma \cdot \vec{\nabla} v_{n}-\gamma \Delta v_{n}+2 \lambda q \gamma v_{n}, \varphi\right\rangle
\end{aligned}
$$

and, by using (10),

$$
\begin{aligned}
\left(\gamma v_{n}, \varphi\right)_{Q}= & -\left\langle v_{n},(\Delta \gamma) \varphi\right\rangle+2\left\langle v_{n}, \vec{\nabla} \cdot[(\vec{\nabla} \gamma) \varphi]\right\rangle \\
& +\left\langle g_{n},(\vec{\sigma} \cdot \vec{D} \gamma) \varphi\right\rangle+\left\langle g_{n}, \gamma(\vec{\sigma} \cdot \vec{D}) \varphi\right\rangle \\
& +\left\langle\lambda f_{n}+\lambda^{2} v_{n}, \gamma \varphi\right\rangle .
\end{aligned}
$$


Therefore we can find a positive constant $C$ independent of $\varphi$ such that

$$
\left|\left(\gamma v_{n}, \varphi\right)_{Q}\right| \leq C\left(\left\|f_{n}\right\|_{\mathbf{h}}+\left\|g_{n}\right\|_{\mathbf{h}}+\left\|v_{n}\right\|_{\mathbf{h}}\right) \cdot\|\varphi\|_{Q} .
$$

Noting that $\mathcal{D}^{2}$ is dense in $\mathbf{h}$, we have $v_{n} \in \mathbf{h}_{Q}$ and

$$
\left\|\gamma v_{n}\right\|_{Q} \leq C\left(\left\|f_{n}\right\|_{\mathbf{h}}+\left\|g_{n}\right\|_{\mathbf{h}}+\left\|v_{n}\right\|_{\mathbf{h}}\right) \quad(n=1,2, \cdots) .
$$

Since $\left\{v_{n}\right\},\left\{f_{n}\right\}$ and $\left\{g_{n}\right\}$ are bounded sequences in $\mathbf{h}$, we select a subsequence $\left\{v_{n_{j}}\right\}_{j=1,2, \cdots}$ of $\left\{v_{n}\right\}$, which is strongly convergent in $\mathbf{h}$, using again Reed-Simon [RS], Thoerem XIII.65. Since $\left\{u_{n}\right\}$ is orhonormal, $\left\{v_{n}\right\}$ converges weakly to 0 in $\mathbf{h}$. Therefore we have

$$
v_{n_{j}} \longrightarrow 0 \text { as } j \longrightarrow \infty
$$

strongly in $\mathbf{h}$. The above inequality (11) and

$$
\left\|f_{n}\right\|_{\mathbf{h}}+\left\|g_{n}\right\|_{\mathbf{h}} \longrightarrow 0 \text { as } n \longrightarrow \infty
$$

in view of $(7)$, yield

$$
\gamma(\vec{\sigma} \cdot \vec{D}) v_{n_{j}}=(\vec{\sigma} \cdot \vec{D})\left(\gamma v_{n_{j}}\right)-(\vec{\sigma} \cdot \vec{D} \gamma) v_{n_{j}} \longrightarrow 0
$$

strongly in $\mathbf{h}$, . By means of (9) we have

$$
\gamma w_{n_{j}} \longrightarrow 0 \text { as } j \longrightarrow \infty
$$

strongly in $\mathbf{h}$. Since $\left\{w_{n}\right\}$ is locally strongly convergent in $\mathbf{h}$, the above property implies the strong convergence of $\left\{w_{n_{j}}\right\}$ in $\mathbf{h}$. Moreover, since it converges weakly to 0 in $\mathbf{h}$, we have

$$
w_{n_{j}} \longrightarrow 0 \text { as } j \longrightarrow \infty
$$

strongly in $\mathbf{h}$. Thus, (12) and (13) give a contradiction to

$$
\left\|u_{n_{j}}\right\|^{2}=\left\|v_{n_{j}}\right\|^{2}+\left\|w_{n_{j}}\right\|^{2}=1, \quad j=1,2, \cdots .
$$

\section{Proof of Proposition 3 and Remarks}

We show first the non-existence of eigenvalues of $H$ in $\mathbf{R}^{-}$. Suppose

$$
\lambda \leq 0, \quad u=\left(\begin{array}{c}
v \\
w
\end{array}\right) \in D(H) \quad(v, w \in \mathbf{h}) \text { and } H u=\lambda u .
$$

Then we have

$$
(\vec{\sigma} \cdot \vec{D}) w+2 q v=\lambda v,
$$




$$
(\vec{\sigma} \cdot \vec{D}) v=\lambda w .
$$

Therefore, $v$ satisfies

$$
-\Delta v+2 \lambda q v=\lambda^{2} v
$$

It is well known that if $\lambda<0$, the Schödinger operator $-\Delta+2 \lambda q(x)$ has no eigenfunctions in $L^{2}\left(\mathbf{R}^{3}\right)$ under the conditions (c-1) and (c-2) (see, e.g., Uchiyama-Yamada [UY]). If $\lambda=0$, we obtain from (15) that $\Delta v=0$. Therefore, $v \in \mathbf{h}$ means $v=0$, which and (14) imply $w=0$ and $u=0$.

Finally, we prove $\mathbf{R}^{-} \subset \sigma(H)$. The proof is given along the same line of Arai-Yamada [AY]. Let us denote

$$
\begin{aligned}
B_{R} & =\left\{x \in \mathbf{R}^{3}|| x \mid \leq R\right\}, \\
E_{R} & =\left\{x \in \mathbf{R}^{3}|| x \mid \geq R\right\}, \\
\Omega & =B_{R / 2},
\end{aligned}
$$

where $R$ is the number in the assumption (c-2), and take a $C^{\infty}$ function $\rho(x)$ such that

$$
\rho(x)=0 \quad(x \in \Omega) \text { and } \rho(x)=1 \quad\left(x \in E_{R}\right) .
$$

Let $\tilde{H}$ be a selfadjoint operator in $\mathcal{H}$ such that

$$
\tilde{H}=(\vec{\alpha} \cdot \vec{D})+\rho(x) q(x)(\beta+I) .
$$

Since the essential spectrum $\sigma_{\text {ess }}(H)$ of $H$ coincides with $\sigma_{\text {ess }}(\tilde{H})$ of $\tilde{H}$, it suffices to prove $\mathbf{R}^{-} \subset \sigma_{e s s}(\tilde{H})$. Let $\left\{\mu_{0}, \mu_{1}, \cdots\right\}$ be the totality of eigenvalues of $-\Delta_{\mid \Omega}$ with Neumann boundary condition, and $\left\{\varphi_{0}, \varphi_{1}, \cdots\right\}$ the corresponding complete orthonormal system of the eigenfunctions such that

$$
0=\mu_{0} \leq \mu_{1} \leq \cdots \text { and } \varphi_{0}(x) \equiv[\operatorname{vol}(\Omega)]^{-1 / 2} .
$$

We show below

$$
\mathbf{R}^{-} \backslash\left\{-\sqrt{\mu_{1}},-\sqrt{\mu_{2}}, \cdots\right\} \subset \sigma(\tilde{H}),
$$

which yields $\mathbf{R}^{-} \subset \sigma_{e s s}(\tilde{H})$. Assume that a negative number $\lambda$ such that

$$
\lambda^{2} \in \mathbf{R}^{+} \backslash\left\{\mu_{1}, \mu_{2}, \cdots\right\} .
$$

would not belong to $\sigma(\tilde{H})$, that is, $\lambda$ would belong to the resolvent set of 
$\tilde{H}$. Then, for

$$
f(x)={ }^{t}\left(\varphi_{0}, \varphi_{0}\right) \quad(x \in \Omega) \text { and } f(x)=0 \quad(x \notin \Omega)
$$

we can find a unique solution $u={ }^{t}(v, w) \in D(\tilde{H}) \subset \mathcal{H}_{l o c}^{1}=\left[H_{l o c}^{1}\right]^{4}$ such that

$$
(\tilde{H}-\lambda) u=\left(\begin{array}{c}
f(x) \\
0
\end{array}\right) .
$$

Then we have

$$
\begin{aligned}
& (\vec{\sigma} \cdot \vec{D}) w+2 \rho(x) q(x) v(x)-\lambda v(x)=f(x), \\
& (\vec{\sigma} \cdot \vec{D}) v-\lambda w(x)=0 .
\end{aligned}
$$

and

$$
-\Delta v+2 \lambda \rho(x) q(x) v(x)-\lambda^{2} v(x)=\lambda f(x) \quad\left(x \in \mathbf{R}^{n}\right) .
$$

and, therefore, $v \in\left[H_{l o c}^{2}\right]^{2}$. In view of Sobolev's theorem (Sobolev [So], p.85) $v(r \cdot)$ and $\frac{\partial v}{\partial r}(r \cdot)$ are strongly continuous in $\left[L^{2}\left(S^{2}\right)\right]^{2}$ with respect to $r>0$. The conditions $(\mathrm{c}-1)$ and $(\mathrm{c}-2)$ gives that $-\Delta+2 \lambda q$ has no eigenfunctions in $L^{2}\left(E_{R}\right)$ without any restriction of boundary conditions (see, e.g., Uchiyama-Yamada $[\mathrm{UY}])$. Therefore, we have $v(x) \equiv 0$ in $E_{R}$. By the unique continuation property of elliptic operators (e.g., EasthamKalf [EK], $\S 6.5$, Corollary 6.5.1), we have $v(x) \equiv 0$ in $E_{R / 2}$ and

$$
-\Delta v-\lambda^{2} v(x)=\lambda\left(\begin{array}{l}
\varphi_{0} \\
\varphi_{0}
\end{array}\right) \text { in } \Omega, v=0 \text { and } \frac{\partial v}{\partial r}=0 \text { on } \partial \Omega .
$$

Since each component of $v$ satisfies the Neumann condition on $\partial \Omega$ as seen above, $v$ can be expanded with $\left\{\varphi_{j}\right\}_{j=1,2, \ldots .}$ Noting $\lambda^{2}$ is none of eigenvalues $\left\{\mu_{j}\right\}$, we have

$$
v(x)=-\frac{1}{\lambda}\left(\begin{array}{c}
\varphi_{0} \\
\varphi_{0}
\end{array}\right) \text { in } \Omega
$$

which contradicts to $v=0$ on $\partial \Omega$.

Remark 1. In Theorem 1 the discrete spectrum $\sigma_{d}(H)$ is unbounded above and below. This is proved as follows. For example, assume $\sigma_{d}(H)$ would be bounded above. Then there exists a positive constant $M$ such that

$$
(H u, u) \leq M\|u\|^{2} \quad u \in D(H) .
$$


Write $u={ }^{t}(v, w) \in \mathbf{h} \times \mathbf{h}$. Then we obtain

$$
\begin{gathered}
2 \operatorname{Re}\langle(\vec{\sigma} \cdot \vec{D}) v, w\rangle+2\langle(p+q) v, v\rangle+2\langle(p-q) w, w\rangle \\
\leq M\left(\|v\|_{\mathbf{h}}^{2}+\|w\|_{\mathbf{h}}^{2}\right) .
\end{gathered}
$$

Since $p(x)$ and $q(x)$ are locally bounded functions, we can find a positive constant $C$ such that

$$
2 \operatorname{Re}\langle(\vec{\sigma} \cdot \vec{D}) v, w\rangle \leq C\left(\|v\|_{\mathbf{h}}^{2}+\|w\|_{\mathbf{h}}^{2}\right) \quad v, w \in\left[C_{0}^{\infty}\left(B_{1}\right)\right]^{2} .
$$

Substituting $w=v$ and $w=-v$ in (16), we have

$$
|\langle(\vec{\sigma} \cdot \vec{D}) v, v\rangle| \leq C\|v\|_{\mathbf{h}}^{2}, \quad v \in\left[C_{0}^{\infty}\left(B_{1}\right)\right]^{2}
$$

which implies that $\vec{\sigma} \cdot \vec{D}$ in $B_{1}$ with Dirichlet boundary condition is a bounded operator in $\mathbf{h}$, that is,

$$
\|(\vec{\sigma} \cdot \vec{D}) v\|_{\mathbf{h}}=\|\nabla v\|_{\mathbf{h}} \leq C\|v\|_{\mathbf{h}}, \quad v \in\left[C_{0}^{\infty}\left(B_{1}\right)\right]^{2} .
$$

This is a contradiction.

Similarly, we obtain that $H$ in Theorem 2 has the discrete spectrum unbounded in $\mathbf{R}^{+}$.

Remark 2. The conditions in Proposition 3 can be weakened. For the nonexistence theorem of eigenvalues of Schrödinger operators plays an important role in Proposition 3. The non-existence theorem for Schrödinger operators has been studied extensively by many authors (see, e.g., the reference of [UY], where the reader can find some works concerning the non-existence theorem.)

It is conjectured in Proposition 3 that the half line $\mathbf{R}^{-}$is included in the absolutely continuous spectrum $\sigma_{a c}(H)$.

Acknowledgment The author would like to thank Prof. M. Arai and the referee for their valuable advices.

\section{References}

[AY] Arai M. and Yamada O., A remark on essential spectrum of magnetic Schrödinger operators with exploding potentials. Memo. Inst. Sci. Engi. Ritsumeikan Univ. 50 (1991), 21-32.

[EK] Eastham M.S.P. and Kalf H., Schrödinger-type Operators with Continuous Spectra. Research Notes in Math. 65, Pitman Publishing, Boston-London-Melbourne, 1982. 
[E] Erdélyi A., Note on a paper of Titchmarsh. Quart. J. Math. Oxford (2) 14 (1963), $147-152$.

[IMS] Ikhdair S., Mustafa O. and Sever R., Solution of Dirac equation for vector and scalar potentials and some applications. Hadronic J. 16 (1993), 57-74.

[J] Jörgens K., Perturbation of the Dirac operator. Lecture Notes in Math. 280, Springer Verlag, Berlin-Heidelberg-New York, 1972, 87-102.

[JT] Jena S.N. and Tripati T., Dirac bound state spectra of $Q \bar{Q}, q \bar{q}$, and $Q \bar{q}$ systems. Phys. Rev. D 28 (1983), 1780-1782.

[RH] Ram B. and Halasa R., Dirac equation with a quadratic scalar potential - are quarks confined? Lett. AL Nuovo Cimento 26 (1979), 551-554.

[RS] Reed M. and Simon B., Methods of Modern Mathematical Physics, IV : Analysis of Operators. Academic Press, New York-San Francisco-London, 1978.

[Sc] Schmidt K.M., Absolutely continuous spectrum of Dirac systems with potentials infinite at infinity. to appear in Math. Proc. Camb. Phil. Soc.

[So] Sobolev S.L., Applications of Functional Analysis in Mathematical Physics. Translations of Mathematical Monographs 7, AMS, Providence, 1963.

[Th] Thaller B., The Dirac Equation. Texts and Monographs in Physics, Springer Verlag, Berlin-Heidelberg-New York, 1992.

[Ti] Titchmarsh E.C. On the nature of the spectrum in problems of relativistic quantum mechanics. Quart. J. Math. Oxford (2) 12 (1961), 227-240.

[UY] Uchiyama J. and Yamada O., Sharp estimates of lower bounds of polynomial decay order of eigenfunctions. Publ. RIMS, Kyoto Univ. 26 (1990), 419-449.

Department of Mathematics

Ritsumeikan University

Kusatsu, Shiga 525-77, Japan

E-mail: yamadaos@bkc.ritsumei.ac.jp 